\title{
Intelligent Optical Systems using Adaptive Optics (Invited)
}

\author{
Natalie Clark, NASA Langley Research Center, Hampton VA 23681
}

\begin{abstract}
Until recently, the phrase adaptive optics generally conjured images of large deformable mirrors being integrated into telescopes to compensate for atmospheric turbulence. However, the development of smaller, cheaper devices has sparked interest for other aerospace and commercial applications. Variable focal length lenses, liquid crystal spatial light modulators, tunable filters, phase compensators, polarization compensation, and deformable mirrors are becoming increasingly useful for other imaging applications including guidance navigation and control (GNC), coronagraphs, foveated imaging, situational awareness, autonomous rendezvous and docking, non-mechanical zoom, phase diversity, and enhanced multi-spectral imaging. The active components presented here allow flexibility in the optical design, increasing performance. In addition, the intelligent optical systems presented offer advantages in size and weight and radiation tolerance.
\end{abstract}

\section{INTRODUCTION}

Active optic devices are quickly finding their way into advanced optical systems for many aerospace applications. NASA Langley Research Center (LaRC) is at the forefront of development and application of these devices and systems and recently constructed and outfitted a new cleanroom at LaRC dedicated to the fabrication of devices and systems. The active optic elements described in this paper rely on birefringent materials to alter either the phase or the polarization of the incoming wavefront. Section 2 describes phase spatial light modulators using birefringent materials. In particular, birefringent liquid crystals for electrically controlled spatial light modulators are described. In section 3 , a novel method for polarization spatial light modulation is presented along with experimental results. In this paper we describe the design and performance of phase and polarization modulation devices for several aerospace applications including a star tracker system, coronagraph, optical communications, and large space telescope systems.

\section{LIQUID CRYSTAL PHASE SPATIAL LIGHT MODULATORS}

Liquid crystal is a state of matter that is intermediate between a crystalline solid and an amorphous liquid. It may also be viewed as a liquid that has an ordered arrangement of molecules such that a molecular orientation exists. Liquid crystals arise under certain conditions in organic (hydrocarbon) chemicals so that the molecules are sharply anisotropic, that is, they have highly elongated (rod-like) molecules or flattened (disc-like) molecules. A direct consequence of the ordering of the anisotropic molecules is the anisotropy of the mechanical, electric, magnetic, and optical properties ${ }^{1-5}$.

\subsection{Array of liquid crystal cells}

NASA LaRC has developed a 1024 x 768 array liquid crystal device with a metalized silicon backplane (commercially obtained) shown below in Figure 1. Such a device is often referred to as a liquid crystal on silicon (LCOS) device. The LCOS panel is basically an electronically controlled phase shifter device with the rubbing direction along the y axis as shown in Figure 1. A previous paper ${ }^{1}$ describes the design, fabrication, and performance evaluation of the liquid crystal devices including a 1024x768 pixelated LCOS (Liquid Crystal on Silicon) device. Table 1 summarizes the performance of the 1024 x 768 LCOS device.

\subsection{Phase shifting calibration of the LCOS array}

The LCOS device was calibrated using a Zygo Interferometer. A schematic of the experimental layout is shown in Figure 2. A sequence of phase maps for each pixel at each gray level was acquired. The non-uniformity was less than $1 / 10$ of a wavelength (lambda); hence, a simple polynomial interpolation of the phase, Y, can be used to represent the pixel controller plant model: 


$$
\mathrm{Y}=\mathrm{p} 1{ }^{*} \mathrm{x}^{\wedge} 4+\mathrm{p} 2 * \mathrm{x}^{\wedge} 3+\mathrm{p} 3 * \mathrm{x}^{\wedge} 2+\mathrm{p} 4 * \mathrm{x}+\mathrm{p} 5
$$

For our LCOS device $\mathrm{p}_{1}=-82.521 ; \mathrm{p}_{2}=425.35 ; \mathrm{p}_{3}=-484.04 ; \mathrm{p}_{4}=-49.964$ and $\mathrm{p}_{5}=213$. Any requirement for increased wavefront accuracy is beyond the capability of commercial interferometers and new more sophisticated wavefront sensors would need to be developed.

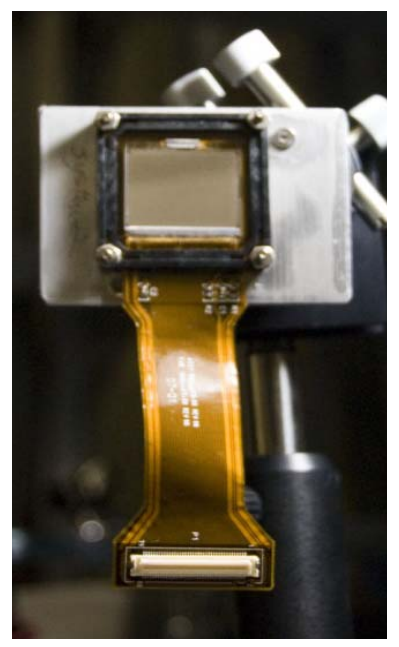

Figure 1. Array of phase shifters. The array of phase shifters consists of a 1024 x 768 array of pixels. Each pixel is a single layer phase shifter.

Table 1. Liquid crystal on silicon (LCOS) device

\begin{tabular}{|l|l|}
\hline Cell Type & Electronic controlled Phase Shifter \\
\hline Active Area & $20 \mathrm{~mm} \times 15 \mathrm{~mm}$ \\
\hline Resolution & $1024 \times 768$ \\
\hline Fill Factor & $96.0 \%$ \\
\hline Bit Depth & 8 bit $(256$ gray scale $)$ \\
\hline Temporal Bandwidth & $60 \mathrm{~Hz}$ \\
\hline Effective Stroke Length & $635 \mathrm{~nm}$ \\
\hline Liquid Crystal Layer Uniformity & $1 / 10$ lambda Peak to Valley at $632.8 \mathrm{~nm}$ \\
\hline Operation Wavelength & $632.8 \mathrm{~nm}$ \\
\hline Beam Steering Range & $+/-4$ mrad on both X and Y axis \\
& Steering Efficiency $>80.3 \%$ \\
\hline Steering Accuracy & Better than $10 \mu$ rad \\
\hline
\end{tabular}




\subsection{Near field performance evaluation of the LCOS array}

Shown in Figure 3 is one frame of the interferogram taken by the Zygo interferometer. Referring to the interferogram, the wavefront distortion between any bright fringes is 1 wavelength at $623.8 \mathrm{~nm}$, or $1 / 2$ wavelength of the surface height difference. The whole device is not a flat surface but has a large spherical shape. The main reason for this is the whole cell is glued to a metal heat sink, which is not flat. When the glue hardens, some tension is formed and thus bends the entire cell. Fortunately, this bending is spherical and simply focusing the optics to compensate for the defocus induced by the device bending results in a very flat wavefront in the optic exit pupil. The liquid crystal device was placed on a micrometer stage capable of moving along the optic axis. The phase shift profiles were recorded by the interferometer. Assuming the irradiance on a given pixel is $\mathrm{I}_{1}(\mathrm{x}, \mathrm{y}), \mathrm{I}_{2}(\mathrm{x}, \mathrm{y}), \mathrm{I}_{3}(\mathrm{x}, \mathrm{y}), \mathrm{I}_{4}(\mathrm{x}, \mathrm{y})$, and $\mathrm{I}_{5}(\mathrm{x}, \mathrm{y})$ for each of the five phase shift stage positions, the wavefront phase profile is given by

$$
\Phi=\tan ^{-1}\left(2\left(\mathrm{I}_{4}-\mathrm{I}_{2}\right) /\left(2 * \mathrm{I}_{3}-\mathrm{I}_{1}-\mathrm{I}_{5}\right)\right) / 2
$$

\subsection{Far field characterization}

The far field beam quality was also measured to characterize the LCOS device. A small telescope with effective focal length of $1170 \mathrm{~mm}$ and a diameter of $76.2 \mathrm{~mm}$ was used. The $650 \mathrm{~nm}$ wavelength laser beam was reflected off the LCOS device and focused. The irradiance profile was measured with a CCD camera. Figure 4 shows the beam profile of the focused laser beam. In order to evaluate the LCOS compensation for the far field, the beam was expanded to form a $1 / \mathrm{e}^{2}$ waist of $1.02 \mathrm{~mm}$ to $1.02 \times 150 / 16.5=9.27 \mathrm{~mm}$. The beam was then focused and measured to have a beam waist of $\mathrm{w}=$ 107.3 microns.

The Strehl ratio is a useful metric to characterize the compensation performance. Figure 5 shows an uncompensated and compensated beam. The Strehl ratio is defined as the normalized peak intensity to diffraction focus. The Strehl ratio of the compensated beam was about 100 times higher than the non-compensated beam. Ideally a diffraction limited compensation would yield a Strehl ratio larger than 0.84. One of the main reasons the LCOS is not quite diffraction limited was because the aluminum mirror on the panel did not have a dielectric coating which resulted in a low reflectivity of $80.4 \%$. Note that the Strehl ratio is normalized to the total energy to measure the percent energy contained in the Airy pattern ${ }^{6}$. The beam waist after beam expansion was $9.27 \mathrm{~mm}$, compared with the $20 \mathrm{x} 15 \mathrm{~mm}$ aperture of the LCOS device. The long axis of the LCOS device is always defined as the Y axis and the short axis is defined as the $\mathrm{X}$ axis. The beam waist after beam expansion was $9.27 \mathrm{~mm}$, which means the entire beam is contained within the aperture of the LCOS device of $20 \times 15 \mathrm{~mm}$. The long axis of the LCOS device is always defined as the Y axis and the short axis is defined as the $\mathrm{X}$ axis.

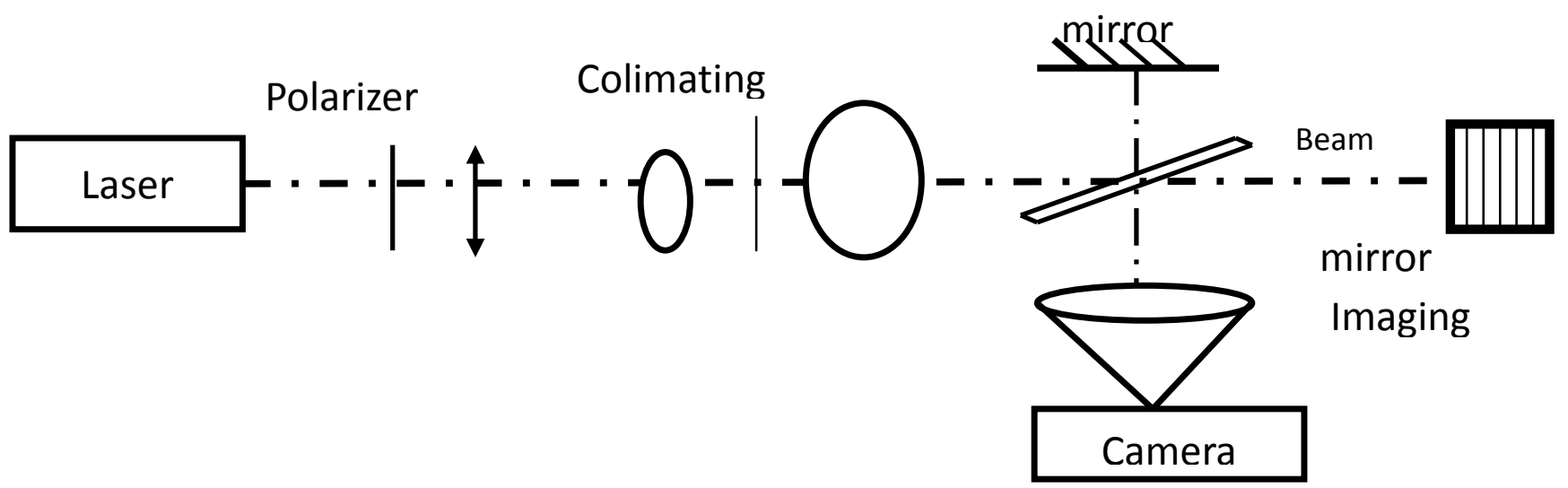

Figure 2. Schematic of the Interferometer test set up. 


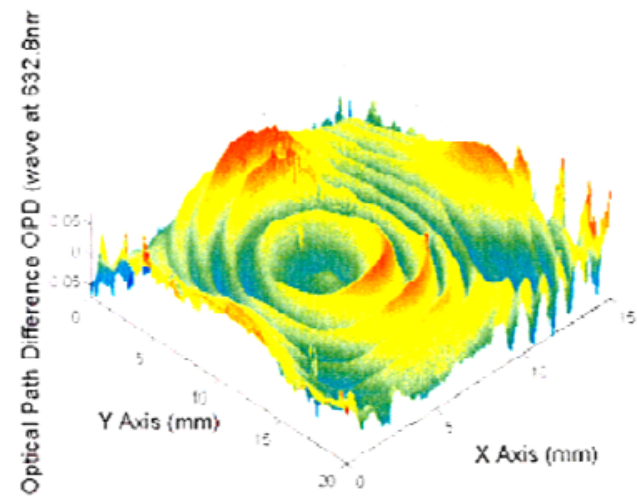

(a)

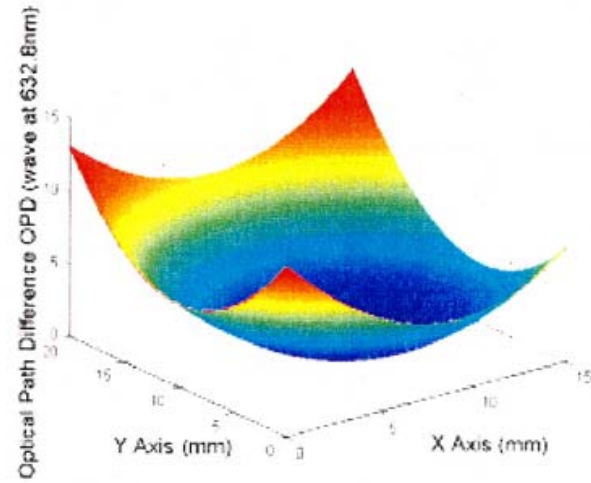

(b)

Figure 3. Interferogram of the LCOS device before and after compensation of defocus aberration. (a) is the wavefront of a defocus aberration introduced by moving the collimating lens to induce a small wavefront aberration of defocus. (b) Surface map of the wavefront residual aberrations after compensation.

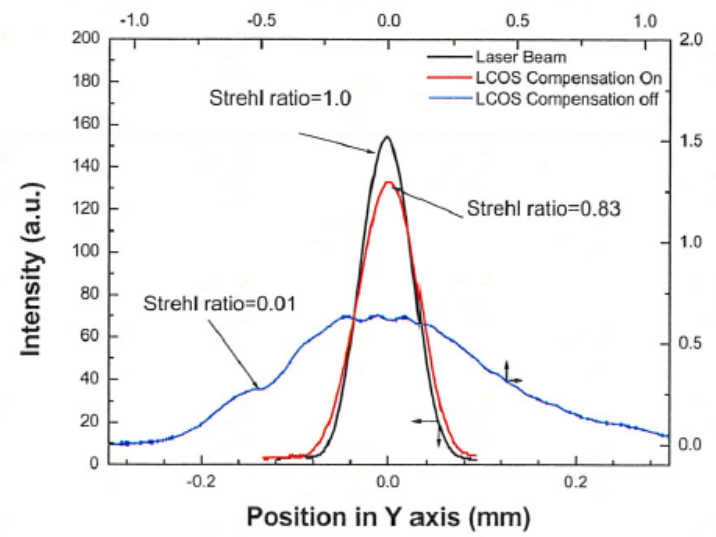

Figure 4. Beam profile of a focused laser beam. The laser beam is shown in black, the LCOS with compensation on is shown in red and the uncompensated laser beam is shown in blue.
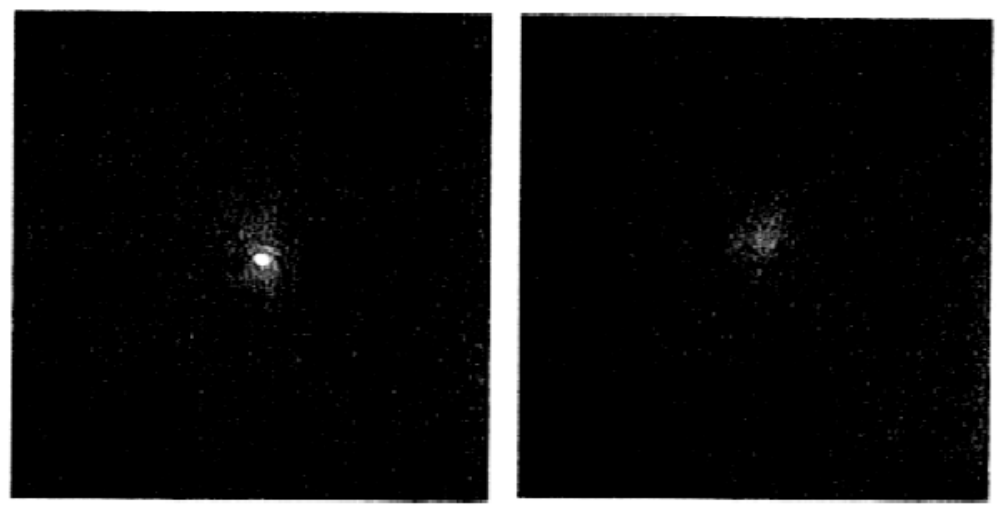

Figure 5. Compensated Beam using LCOS device. (a) compensated beam using the LCOS device as a phase modulator (b) uncompensated beam. 


\section{POLARIZATION COMPENSATION}

Direct imaging of terrestrial exoplanets requires a telescope/instrument capable of controlling and suppressing unwanted star light to approximately one part in $10^{12}$ over a wide wavelength bandpass spanning the UV to near IR spectrum ${ }^{7-12}$. Terrestrial exoplanets are faint requiring large aperture telescopes to record their image. Because of the required size of the aperture (3 to 8 meters), coronagraph designs that fit into launch vehicles employ low F\# (typically 1 to 1.5 ) to collect and focus the radiation. These low F\# systems require a steeply curved mirror that introduces spatially varying polarization effects which in turn reduce system transmittance and increase scattered light to unacceptable levels.

The properties of metal and dielectric anisotropic coatings have been largely overlooked in the analysis and performance characterization of low F\# telescopes for exoplanet missions, ${ }^{7,11}$. Several coronagraph architectures have been proposed $^{7-13}$. Each one of these will have degraded performance because of Fresnel polarization apodization. This section addresses the modeling, and mitigation of Fresnel polarization effects in space-based coronagraphic telescopes using an innovative device that incorporates birefringent nanolayers ${ }^{14}$ to control the amplitude and phase of polychromatic white light wavefronts. The polarization compensation device, which we refer to as the Spatially Varying Retarder Plate (SVRP) uses broadband white-light optical polarization and retardation control to maximize coronagraph system transmittance, contrast, and to minimize losses to scattered light. As discussed in Section 3.1, this device can be located at a much smaller pupil plane ( $\sim 10$-cm diameter) which will be much smaller, lighter, and easier to fabricate than an optical element located on or next to the primary mirror. An understanding of the sources of partial polarization in full-scale coronagraphs is necessary for accurate performance modeling, specification of mirrors and coatings, and to design necessary alternatives to mitigate the effects of this internal polarization produced by Fresnel effects.

\subsection{Polarization compensation in coronagraph system design considerations}

A schematic of an imaging coronagraph telescope system is shown in Figure 6. Referring to the figure, the chief ray is dotted and the marginal ray is dashed. The location where the marginal ray crosses the optic axis is an image plane. The location where the chief ray crosses the optic axis is the pupil plane (an image of the pupil). As with most astronomical telescopes the entrance pupil is co-located with the telescope aperture. Powered optical elements are shown at planes 1, 3 and 5. The ring mask, shown at plane 4 is not a powered element. Although the telescope primary may be quite large, 8 meters as planned in NASA exoplanet missions ${ }^{7-12}$, the mitigation of Fresnel Polarization can be done at a much smaller and convenient pupil plane such as at plane 4 where the ring mask is located. The functions of the polarization compensator and ring mask can be combined, as we are describing under this effort. The diameter of the pupil in the NASA testbed system is on the order of $3 \mathrm{~cm}$. For the final exoplanet missions using $8 \mathrm{~m}$ telescope the ring mask would be much larger. The Langley Research Center Optical Nanomaterials Synthesis and Fabrication Laboratory (ONSL) facilities support fabrication of devices with $50 \mathrm{~nm}$ position resolution, 0.6 micron feature sizes for advanced optical optic devices of even meters in diameter. Because the key fabrication equipment is digitally controlled, devices are fabricated inexpensively and rapidly, facilitating design refinements and modifications. Hence, the facility supports fabrication of polarization compensation devices throughout the exoplanet mission from demonstration in one of the NASA prototype coronagraph systems all the way to the final flight device ${ }^{14}$.

\subsection{Fresnel Polarization Analysis using Extended Jones and Berreman’s 4x4 Method}

Radiation incident on the large primary mirror becomes partially polarized upon reflection. There are several methods used to characterize the polarization in an optical system and optical devices. All of these methods are useful, (or not so useful) depending on specific quantitative applications. Jones calculus ${ }^{4,15-16}$, which characterizes a birefringent network, is a powerful technique in which the state of polarization is represented by a two-component column vector, and each optical element (or layer) is represented by a $2 \times 2$ matrix. The Stokes vector and Muller matrix are based on the same physical approximations as the Jones vector ${ }^{4,14-17}$, but relates to the irradiance the detector sees and is often used in remote sensing applications. The Stokes vector and Muller matrix also include depolarization explicitly in the matrix. Since both Jones and Muller calculus are based on the same physics models, both methods are limited to normally incident and paraxial rays only. For example, the two polarization states of light incident on an anisotropic material (mirror, coating, and SVRP layer) are in general not mutually orthogonal for off axis light. Both the Jones and Muller calculus methods neglect the Fresnel refraction and reflection at the surfaces. These methods do not offer an explanation 
of the light leakage for off axis light. Hence other polarization techniques such as the extended Jones vector, Berreman's $4 \times 4$ matrices, or Finite Difference Time Domain (FTDT) are used instead ${ }^{4,14-25}$.

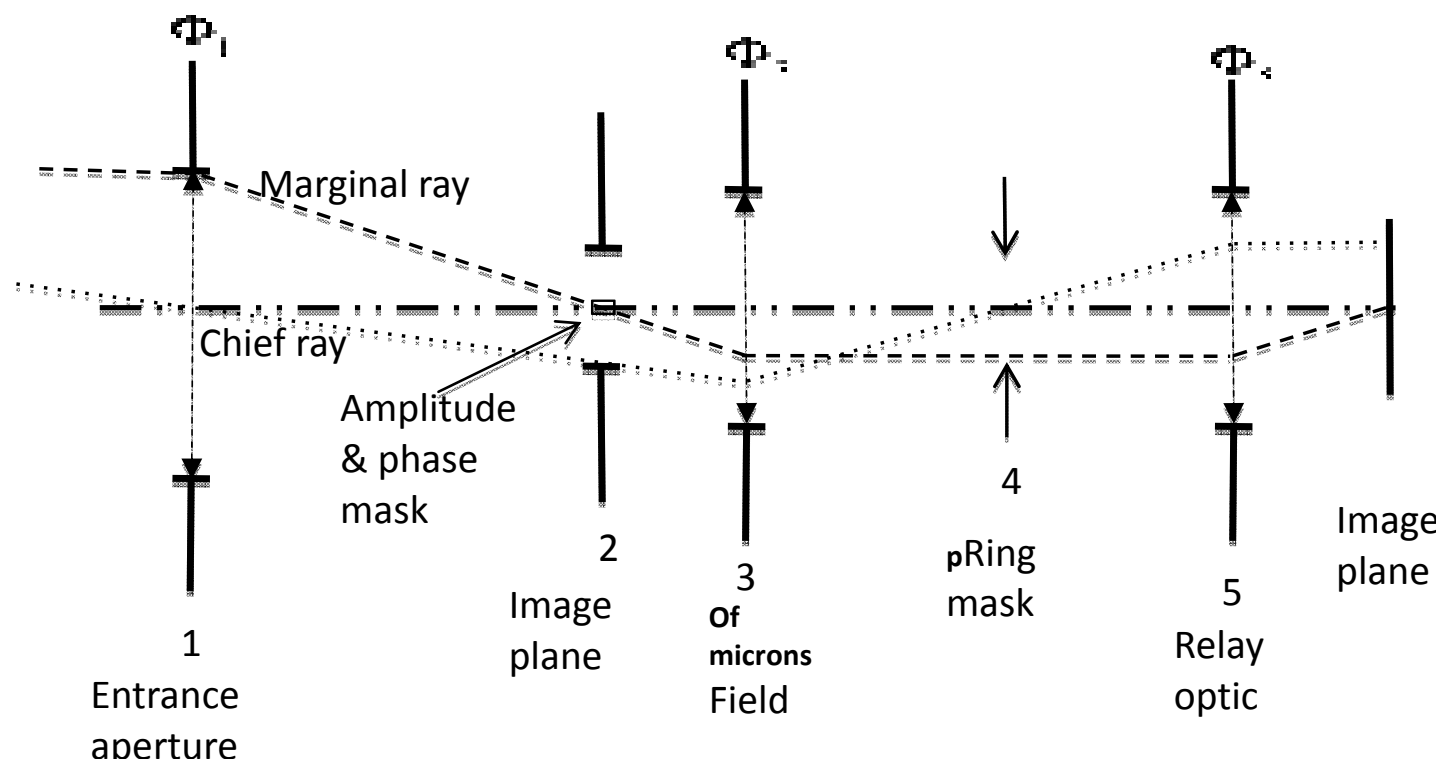

Figure 6. Schematic view of an imaging coronagraph-telescope. The coronagraph is used to control scattered light in optical systems built for high contrast stellar astronomy applications such as exoplanet and binary star atmospheric research.

The Berreman $4 \times 4$ matrix method relates to the propagation of polarized light to stratified media that are uniform in their dielectric properties in one plane, which is taken to be the xy plane. The method is discussed in this section and in more detail in the literature ${ }^{14-22}$. The components of the electric field $\mathrm{E}$ and the magnetic field $\mathrm{H}$ in the plane of the layer can be solved from Maxwell's equations as:

$$
\left[\begin{array}{c}
E_{x}(x, y, z, t) \\
\mu_{0} c H_{y}(x, y, z, t) \\
E_{y}(x, y, z, t) \\
-\mu_{0} c H_{x}(x, y, z, t)
\end{array}\right]=\boldsymbol{\psi}(z) e^{-i \omega\left(t-\frac{\eta x}{c}\right)}
$$

Where $\boldsymbol{\psi}(z)$ is a column vector and where the angular frequency $\omega$ is related to the wavelength in vacuum $\lambda$ and the speed of light $\mathrm{c}$ by:

$$
\omega=c k=\frac{2 \pi c}{\lambda}
$$

Here, $k=2 \frac{\pi}{\lambda}$ is the magnitude of the wave vector in a vacuum. For the sake of convenience, the $\mathrm{x}$ axis is chosen such that the light wave properties in the $\mathrm{xz}$ plane which the plane of incidence. The $\mathrm{x}$ component of the wave vector is equal to $\frac{\eta \omega}{c}=\eta k$, meaning that $\eta$ is proportional to this in plane wave vector component. The column vector $\boldsymbol{\psi}$ satisfies the Berreman equation:

$$
\frac{d \psi}{d z}=i k D \cdot \psi
$$


where $\mathrm{D}$ is a $4 \times 4$ matrix generally referred to as the Berreman matrix (or simply the $4 \times 4$ matrix). Assuming that the magnetic susceptibility can be neglected, the optical properties of the dielectric can be described by the dielectric tensor with components $\epsilon_{\alpha \beta}(\alpha, \beta=x, y, z)$. Then the following expression for the Berreman's matrix $\mathrm{D}$ is

$$
D=\left[\begin{array}{cccc}
-\eta \frac{\epsilon_{z x}}{\varepsilon_{z z}} & 1-\frac{\eta^{2}}{\epsilon_{z z}} & -\eta \frac{\epsilon_{z y}}{\epsilon_{z z}} & 0 \\
\epsilon_{x x}-\frac{\epsilon_{x z} \epsilon_{z x}}{\epsilon_{z z}} & -\eta \frac{\epsilon_{x z}}{\epsilon_{z z}} & \epsilon_{x x}-\frac{\epsilon_{x z} \epsilon_{z x}}{\epsilon_{z z}} & 0 \\
0 & 0 & 0 & 1 \\
\epsilon_{x x}-\frac{\epsilon_{x z} \epsilon_{z x}}{\epsilon_{z z}} & -\eta \frac{\epsilon_{z x}}{\varepsilon_{z z}} \epsilon_{x x} & -\frac{\epsilon_{x z} \epsilon_{z x}}{\epsilon_{z z}} & 0
\end{array}\right]
$$

Equation 3 and 4 are the central equations of the Berreman's $4 \times 4$ matrix method. There are several ways to solve numerically as well as analytically,19 We follow the Eidner-Olano ${ }^{19-22}$ method.

\subsection{Fresnel effects of the telescope primary mirror}

Breckinridge and Oppenheimer ${ }^{7}$ showed that polarization introduced in the telescope-coronagraph system increases scattered light to limit system performance. There are two sources of this unwanted polarization: one is characteristic to the natural curved shape of the primary mirror, which we call the Fresnel polarization. The other is an anisotropic characteristic of the deposition process of the high reflectivity metal coatings required for high efficiency. This anisotropy results in unwanted birefringence, which we call the anisotropy polarization. Spatially non-uniform anisotropies in the deposited thin film introduce unwanted phase and amplitude errors on the wavefront to increase scattered light, degrade image quality and lower contrast. The curvature of the mirror introduces a polarization apodization across the pupil ${ }^{7,14}$ resulting in aberrations, which, in turn also effects the system image quality and lowers contrast.

The Berreman's method can be extended to compute the polarization apodization in terms of contrast and retardance, as a function of angle of incidence $\theta$ and wavelength $\lambda$, across the aperture (or corresponding pupil). The metallic mirror is approximated as a perfect conductor. In such a perfect conductor any electric field coming from external sources will be cancelled by the rearrangement of the freely moving charges. As a consequence the net electric field in the mirror must be zero, and at the interface $\mathrm{E}_{\mathrm{x}}=\mathrm{E}_{\mathrm{y}}=0$.

For real metals the finite conductivity $\sigma$ must be taken into account. As a consequence the dielectric constant $\epsilon$ must be replaced by $\epsilon+\frac{i \sigma}{\epsilon_{0} \omega}$, thus, the refractive index $n=\sqrt{\epsilon+\frac{i \sigma}{\epsilon_{0} \omega}}$ is complex ${ }^{7}$. For sufficiently large $\sigma$ the electric field is inversely proportional to the square root of $\sigma$, which implies that in the limit of a perfectly conducting metal $(\sigma \rightarrow \infty)$ the electric field at the interface is restricted to the case of an ideal metallic mirror. Using this Berreman's extension, we define $S_{1}$ as,

$$
S_{1}=\frac{H}{V}
$$

where $\mathrm{H}$ is the intensity measured for the light polarized horizontally and $\mathrm{V}$ is the intensity measured for the vertically polarized light. Figure 7(a) shows a three dimensional view of a plot of Log to the base 10 of $S_{1}$ as a function of the light beam incidence angle, from zero to thirty degrees onto a tilted thin film aluminum coated mirror and as a function of wavelength. The extended Berreman's 4x4 matrix approach can be used to calculate the retardance as a function of angle of incidence $\theta$ and wavelength $\lambda$ as shown in Figure 7(b). Referring to figure 7(b), the phase angle in degrees on the vertical axis between the $\mathrm{H}$ and the $\mathrm{V}$ components is shown as a function of the incidence angle, from zero to thirty 
degrees on a tilted thin film aluminum coated mirror, and as a function of wavelength. These Figures show the significance of the birefringence and phase on reflection from a highly reflecting metal film. The model agrees very favorably with Breckinridge and Oppenheimer's results ${ }^{7}$ as shown in Figure 7(a). Moreover, the present model provides the retardance induced by the mirror as shown in Figure 7(b). Although these graphs are for an ideal mirror, one can design a compensator for any polarization state and fabricate it in the ONSL ${ }^{14}$.

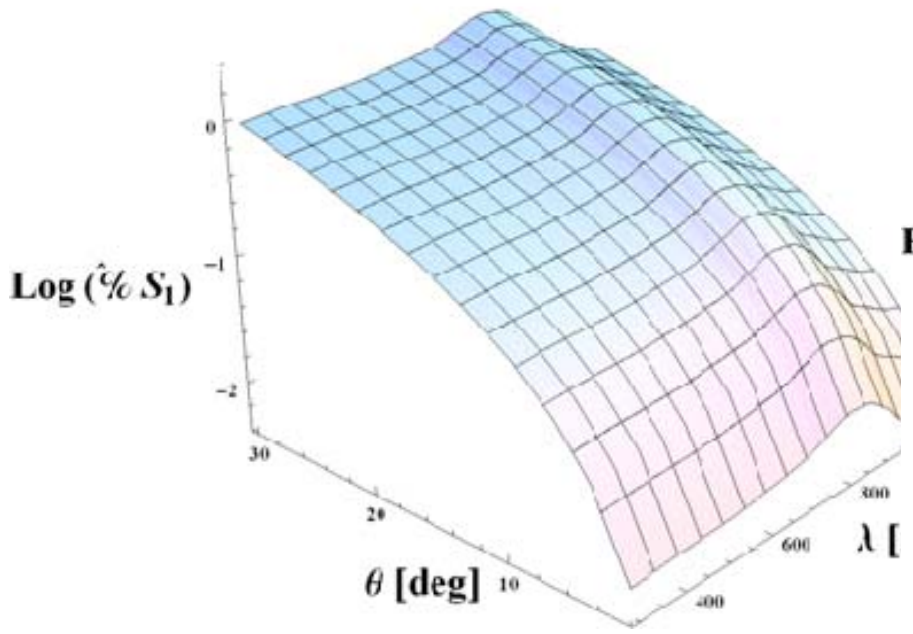

(a)

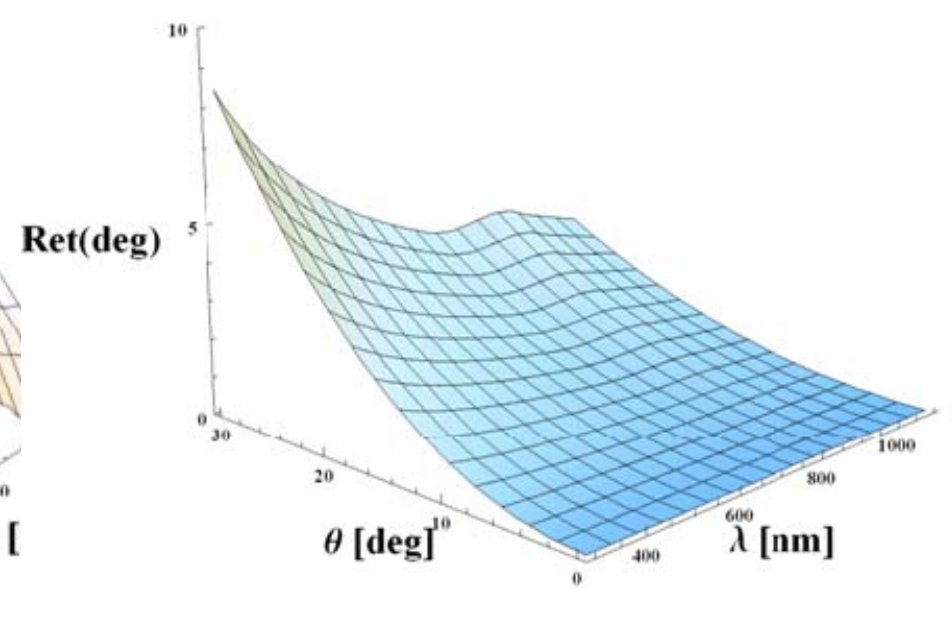

(b)

Figure 7. Polarization contrast (a) and retardance (b) as a function of angle of incidence $\theta$ and wavelength $\lambda$. The plots represent a horizontal "slice" through half of the mirror.

\subsection{Mitigating Fresnel polarization using a spatially varying retardance plate (SVRP)}

A spatially varying retarder plate (SVRP) can be placed at a convenient pupil plane, such as the ring mask plane 4 shown in figure 6. The SVRP consists of a stack of nanolayers of anisotropic and/or isotropic materials $\mathrm{P}_{1}, \mathrm{P}_{2}, \mathrm{P}_{3} \ldots \mathrm{P}_{\mathrm{n}}$ as illustrated in Figure 8. Each layer has it's slow axis oriented at an azimuth angle $\gamma_{1}, \gamma_{2}, \gamma_{3}, \ldots \gamma_{n}$

Optical birefringence describes the difference of a material's refractive index with direction. When the birefringence is on the order of the change of the in-plane refractive index between adjacent materials, surprising and useful optical effects occur.

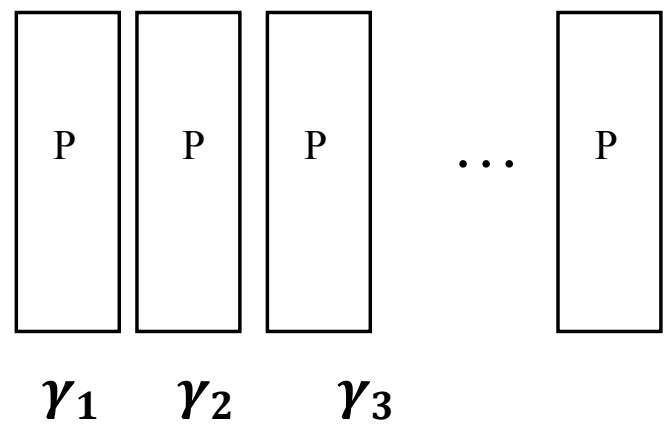

Figure 8. Synthesized Optical material (a). A stack of anisotropic and isotropic material $\mathrm{P}_{1}, \mathrm{P}_{2}, \mathrm{P}_{3} \ldots \mathrm{P}_{\mathrm{n}}$ can be stacked. Each layer thickness, birefringence, and orientation is layered to produce a custom polarization compensator. The SVRP consists of a pixelated array of these stacks as shown in (b). The resolution of each stack can be as small as 0.6 micron and with the appropriate boundary conditions the SVRP can act as a continuous spatially varying sheet or a discrete pixelated device. 
A wide variety of birefringent materials and fabrication methods are being investigated for developing the SVRP device to determine the most suitable to enable $>10^{12}$ contrast. One such material is $\mathrm{MgF}_{2}$. We first consider a single layer $\mathrm{MgF}_{2}$. For multi layers, a device of Quartz is useful. Quartz has a transparency range extending from $0.25 \mu \mathrm{m}$ to $2.5 \mu \mathrm{m}$ $(>99 \%)$ which is a suitable bandwidth of interest in coronagraphs. $\mathrm{MgF}_{2}$ is transparent over an even broader spectral range. Both Quartz and MgF2 are birefringent, but quartz has a much smaller birefringence than MgF2. Hence, these two materials are often used in tandem, for example in the design of achromat waveplates. Both materials are widely used in high-performance optical devices and systems and for coatings. Quartz and MgF2 both are commonly used in high performance optical systems and are known to have high transmittances $>90 \%$ over $300 \mathrm{~nm}$ to $1200 \mathrm{~nm}$. In addition, the very low scattering making them a suitable candidate for the SVRP. Other birefringent materials under consideration include liquid crystals, polyimide, and photo polymers.

\subsection{Optical nanomaterial synthesis design methodology}

To design a SVRP, one must first consider the stack of dielectric (birefringent or isotropic layers) as shown in figure 8. The Berreman's method can be used to compute a propagator matrix $\mathrm{P}_{\mathrm{j}}$ for each layer in the stack. The propagator matrix can be expressed in terms of $\mathrm{D}$ as

$$
P=\exp (i k D)=\sum_{l=0}^{\infty} \frac{(i k d)^{l}}{l !} D^{l}
$$

Where the Taylor series of the exponential function is used to define that function for the matricies. The Matrix D can be written in the diagonal form as

$$
\begin{gathered}
D=T \bar{D} T^{-1} \\
T_{k l}=\psi_{k}^{(l)} \\
\overline{D_{l m}}= \begin{cases}\mu_{l} & \text { if } l=m \\
0 & \text { if } l \neq m\end{cases}
\end{gathered}
$$

Clearly D can be expressed in terms of the eigenvalues of $\mathrm{D}$, whereas $\mathrm{T}$ can be expressed in terms of the eigenvectors of D. Substitution to equation (2) gives:

$$
\begin{gathered}
P=T \bar{P} T^{-1} \\
\bar{P}_{l m}=\left\{\begin{array}{c}
\exp \left(i k \mu_{l} d\right) \text { if } l=m \\
0 \quad \text { if } l \neq m
\end{array}\right.
\end{gathered}
$$

Another relatively recent $4 \times 4$ matrix method for general biaxial media, differing form Berreman's method has been proposed by Yuan and co-workers ${ }^{5,16-18}$. As noted in the literature, the two methods are equivalent, as both are based on the exact solutions to Maxwell's equations. In particular, the quartic equation for the $\mathrm{z}$ component of the wave vector $\mathrm{k}_{\mathrm{z}}$ given by Yuan corresponds to the quartic equation of the eigenvalues of the Berreman matrix $\mu$, because these eigenvalues are equal to $\mathrm{k}_{\mathrm{z}} / \mathrm{k}$.

For the stack of dielectric (birefringent or isotropic) layers shown in Figure 9 (c), each layer is labeled 1,2,3..,N the $4 x 4$ propagation matrix $\mathrm{P}_{\mathrm{j}}$ of each individual layer can be calculated using equation 2 . In order to find the overall propagation matrix $\mathrm{P}$ of the stack of dielectric layers, boundary conditions at the interface between two dielectric layers $\mathrm{m}$ and $\mathrm{n}$ are needed. According to Maxwell theory the components of the electric and magnetic fields $\mathrm{E}$ and $\mathrm{H}$ parallel to the interface between two dielectric medial $\mathrm{m}$ and $\mathrm{n}$ must be continuous, provided that no charge or currents are present at the 
interface. As a consequently $\mathrm{E}_{\mathrm{x}}, \mathrm{E}_{\mathrm{y}}, \mathrm{H}_{\mathrm{x}}, \mathrm{H}_{\mathrm{y}}$ are continuous at the interface, i.e. the column vector $\psi$ satisfies the boundary condition

$$
\begin{gathered}
\boldsymbol{\psi}_{+}=P \cdot \boldsymbol{\psi}_{-} \\
\mathrm{P}=\mathrm{P}_{\mathrm{n}} \ldots \mathrm{P}_{3} \mathrm{P}_{2} \mathrm{P}_{1}
\end{gathered}
$$

Here $\boldsymbol{\psi}_{+}$and $\boldsymbol{\psi}_{-}$are the Berreman column vectors at the two sides of the stack of dielectric layers

\subsection{Design Example of a SVRP}

The design of a spatially varying retarder plate (SVRP), requires careful consideration of the material properties and fabrication techniques. Not only does the birefringence vary spatially across the mirror, but the orientation of the polarization state varies spatially as well, as depicted in figure 9(a). The orientation of the polarization as a function of position is shown in Figure 9 (a). Referring to the figure, two arbitrary small regions P1 and P2 have different birefringence and different polarization orientations. For example the alignment layer can be in correspondence with the polarization orientation of the reflected light off the mirror at a pupil plane as depicted in Figure 9(b). The birefringent stack consisting of layer $\mathrm{B}_{1}, \mathrm{~B}_{2} . . \mathrm{B}_{\mathrm{n}}$, as shown in Figure 9(c). The nano-layers are stacked and the stacking arrangement varies spatially (to a resolution of 0.6 microns for our fabrication process) to yield the desired polarization compensation to mitigate the Fresnel effects. The final isotropic and amplitude layers compensate for the wavefront variation and amplitude variation respectively to yield a uniformly, optically flat, wavefront in a uniform polarization state. Although the design depicted in Figure 9 illustrates the case with the alignment layer is in the direction of the polarization state of the aperture (or pupil), in general the orientation of the alignment layer correspond to the design of the birefringent stack used to compensate for the Fresnel polarization.

Consider the marginal ray of an $\mathrm{F} \#=1$ Telescope that has a 30 degree angle of incidence. This represents the worst case scenario of a telescope system. A 2D plot of the retardance vs wavelength from figure $7(\mathrm{~b})$ is shown if figure $10(\mathrm{a})$. Referring to the figure, the telescope has approximately 9 degrees of maximum retardance (around $300 \mathrm{~nm}$ in the figure) for a 30-degree angle of incidence. The retardance verses wavelength for a 15 degree angle of incidence is shown in Figure 10(b), which has 2 degrees of retardance.

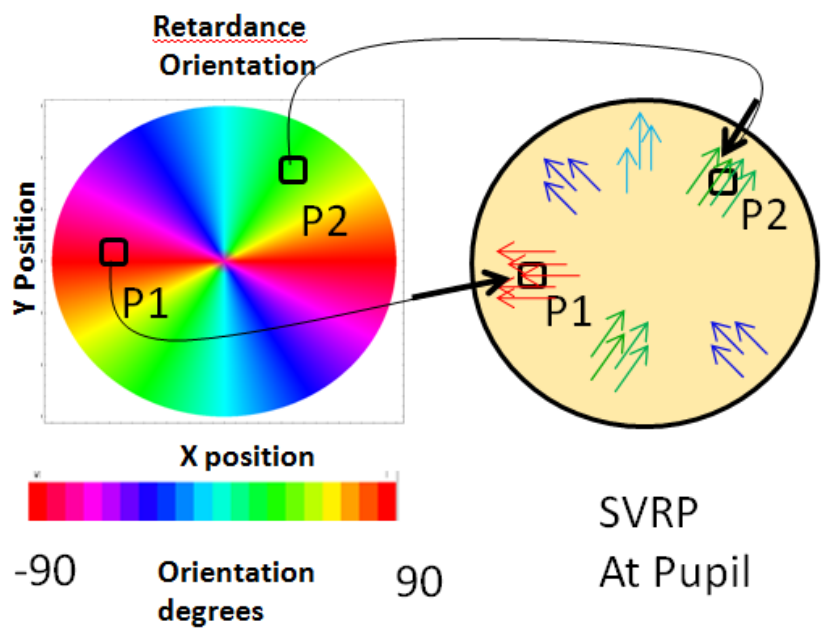

(a)

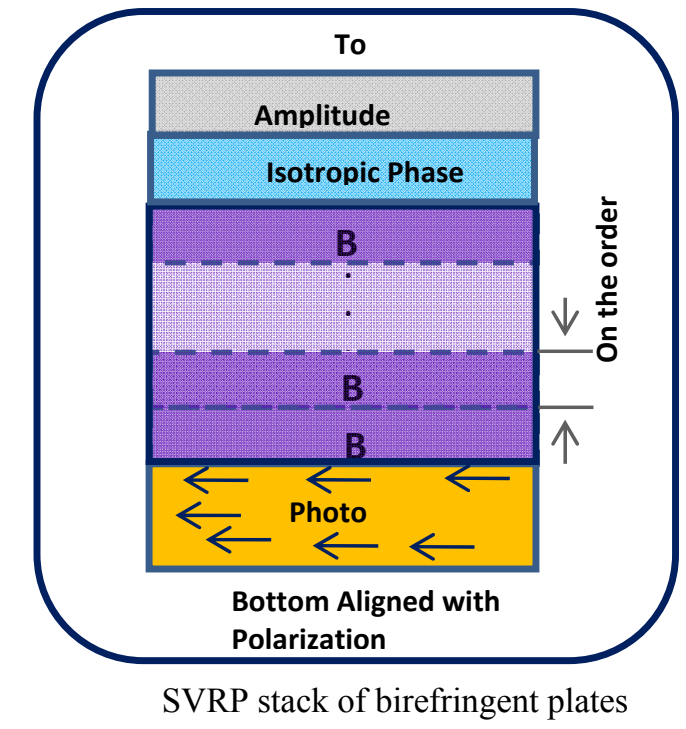

(c)

Figure 9. Photo alignment layer of a SVRP plate. (a) shows a spatially variable retarder plate (SVRP) face on (x,y) with retardance direction indicated by the colors shown in the stripe below which maps color into orientation in degrees as shown. (b) shows two particular regions, P1 and P2 which have two different polarization states and orientation are shown. (c) shows a diagram of the typical stack or sandwich. The bottom layer is a layer of homogeneous dielectric oriented to the 
polarization direction. Birefringent layers of $\mathrm{B}_{1}, \mathrm{~B}_{2} \ldots \mathrm{B}_{\mathrm{n}}$ will be deposited with thickness layers and specific process recipe calculated and optimized to compensate for the Fresnel polarization of light reflected form the telescope.

The contrast $\left(I_{\max }-I_{\min }\right) /\left(I_{\max }+I_{\min }\right)$ of a coronagraph system is related to the diattenuation and retardance by

$$
\text { contrast }=K(1-\cos (\Gamma))
$$

For a simple one-layer SVRP plate the polarization compensation for the 30 degree and 15 degree angle of incidence is shown in Figure 10. Since the dispersion of a nano layer is very small, the polarization compensation is not that dependent on the material selection. Hence, other material will result in comparable polarization compensation as that shown in Figure 11. The material selection is however very important for consideration of clarity, transmission, alignment, fabrication, thermal and space-radiation effects.
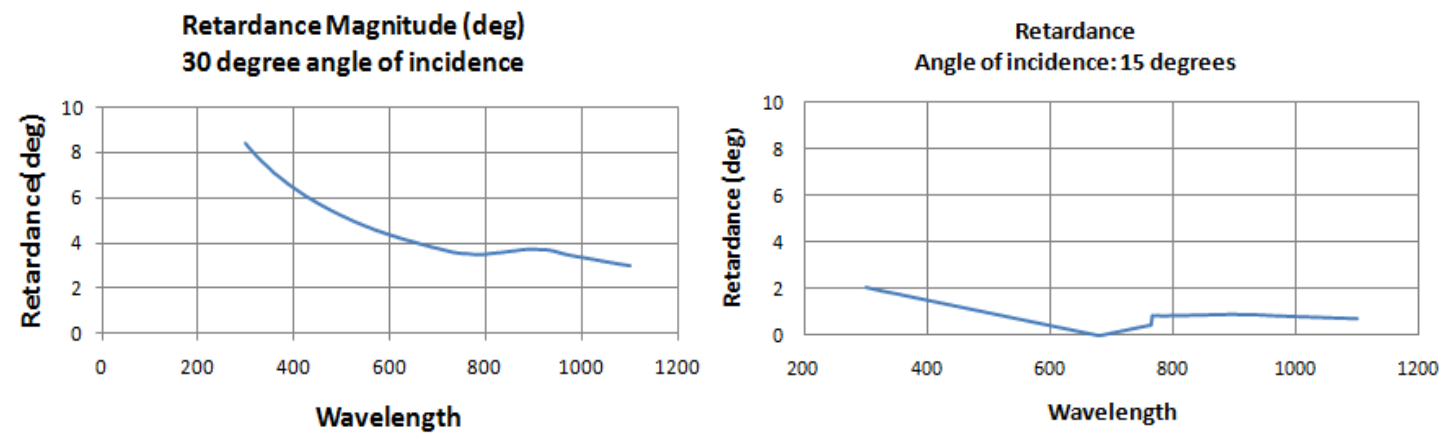

Figure 10. Retardance as a function of wavelength (a) at an angle of incidence 30 degrees (b) at an angle of incidence of 15 degree
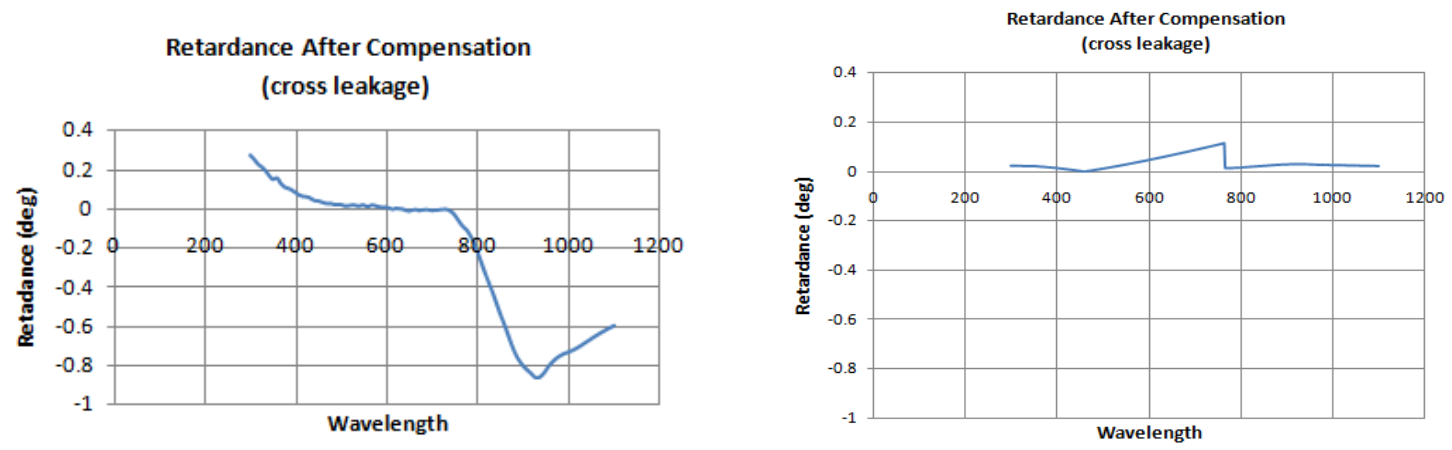

Figure 11. Single cell design of MgF2. (a) For an angle of incidence of 30 degrees retardance as a function of wavelength and after compensation by a single layered device reduces the retardance to better than 0.01 degree over $500-700 \mathrm{~nm}$. Note the scale is $1 / 10$ that of Figure 6. 


\subsection{Fabrication and experimental results.}

The ability of the birefringent layers to orient in a particular direction when in contact with a specially prepared surface is a phenomenon of major importance other SVRP plate fabrication. There are several methods used in the NASA Langley OSNL laboratory. The lab has made significant progress in the development of photo aligned materials. Manufacturers require alignment layers with a wide range of optimized properties. Thin films must easily be formed with high photosensitivity in order to facilitate rapid processing and also to avoid unwanted degradation. The resultant photoaligned film must be insoluble and thermally, electrochemically and photo-chemically stable.

\subsection{Photo-alignment using photo degradation}

The successful photo-alignment of a polyimide is considered to be an important technological goal, because of the high thermal stability of polyimides and their acceptance as the alignment layer of choice by the LC display industry. Hasegawa and Taira ${ }^{22-26}$ first reported photo-alignment of polyimide by polarized light exposure at $257 \mathrm{~nm}$. Homogeneous LC alignment was obtained in a direction perpendicular to the polarization of the incident UV beam. This is the direction of the maximum density of unbroken polyimide chains on exposure. Therefore, alignment was attributed to the anisotropic depolymerization of the polyimide. Further studies confirmed this and the alignment direction was shown to switch by varying the polarization direction of the aligning beam ${ }^{26}$.

For the current work, Nissan SE-7492 in the form of polyamic acid was used. The polyimide film was spin-coated onto a quartz substrate. It was cured at 250 degrees $\mathrm{C}$ for one hour or 200 degrees $\mathrm{C}$ for an hour and a half. A $200 \mathrm{~W} \mathrm{Hg}$ (Xe) lamp equipped with a dichroic mirror reflecting from 230 to $430 \mathrm{~nm}$ was used as the light source. A dichroic UV linear polarizer was used to obtain the linearly polarized UV light. The average power density for the un-polarized and polarized light was approximately 40 and $10 \mathrm{~mW} / \mathrm{cm}^{2}$, respectively, measured using a radiant energy meter.

The transmittance curve from this rudimentary spectroscopic ellipsometer can be processed along with a simple model to determine the dispersion of the birefringence. The standard expression for an $a$-plate with positive birefringence between crossed polarizers applies $^{8-9}$ :

$$
T=\frac{1}{2} T_{0} \sin ^{2}(2 \theta) \sin ^{2}\left(\frac{\pi \Delta n d}{\lambda}\right)
$$

where $T_{0}$ is the unpolarized irradiance incident on the first polarizer, $\lambda$ is the wavelength, $d$ is the film thickness, $\Delta n$ is the birefringence, and $\theta$ is the angle between the effective optical axis of the birefringent film and the transmission axis of either polarizer always set to $45^{\circ}$ in our geometry) Since $T$ is a periodic function involving a square root, the value of $\Delta n$ cannot be unambiguously determined without some additional steps. We have used two methods to find the dispersion of the birefringence from the transmittance. The first method involves a direct computation from the data as follows. From Eq. (13), the birefringence can be expressed as:

$$
\Delta n=\frac{\lambda}{\pi d}\left[m \pi \pm \sin ^{-1}\left(\sqrt{\left.2 T / T_{0}\right)}\right]\right.
$$

Where $\mathrm{m}$ is a non negative integer that describes the order of the solution. From basic principles of these optically transparent materials, we know that $\Delta n$ is continuous across the visible spectrum. Hence, the sign of the $\sin ^{-1}$ function and the order $\mathrm{m}$ are constant between each maximum and minimum in $\mathrm{T}$. Then one can simply manually or computationaly choose both parameters for each data point such that the ambiguity is resolved and a continuous solution $\Delta n(\lambda)$ is found.

A second method that is very useful in optical system design ${ }^{5,14,24-25}$ involves an indirect solution that requires the leastsquares-fit of a dispersion model to the transmittance. Since the polymer films are optically transparent throughout the visible region, the refractive index can be modeled by the first-order Sellmeier dispersion ${ }^{17}$ relation: 
$n(\lambda)^{2}-1=a+b \lambda^{2} /\left(\lambda^{2}-\lambda_{0}^{2}\right)$, where $a, b$, and $\lambda_{0}$ are constants. Granting the standard assumptions, this can be reduced to the slightly simpler Cauchy formula: $n(\lambda)=A+B / \lambda^{2}$, where $A$ and $B$ are constants for a given material. The dispersion of the birefringent films is therefore modeled by the following equation:

$$
\Delta n(\lambda)=n_{e}(\lambda)-n_{o}(\lambda) \approx \Delta n_{\infty}+\frac{C}{\lambda^{2}}
$$

where $\Delta n_{\infty}$ is the birefringence at long wavelengths and $C$ is a constant. Note that the absolute values of the ordinary $(n o)$ and extraordinary $(n e)$ indices are not required to determine $\Delta n(\lambda)$. In order to calculate the dispersion of the birefringence, we combine Eq. (13) and Eq. (14) and utilize least squares minimization to find the parameters $\Delta n_{\infty}$ and $C$ that give the closest fit to our transmittance data. Figure 12 shows the birefringence versus wavelength of a 10 micron polyimide layer in which the optic axis was photo-aligned using our photo-degradation method.

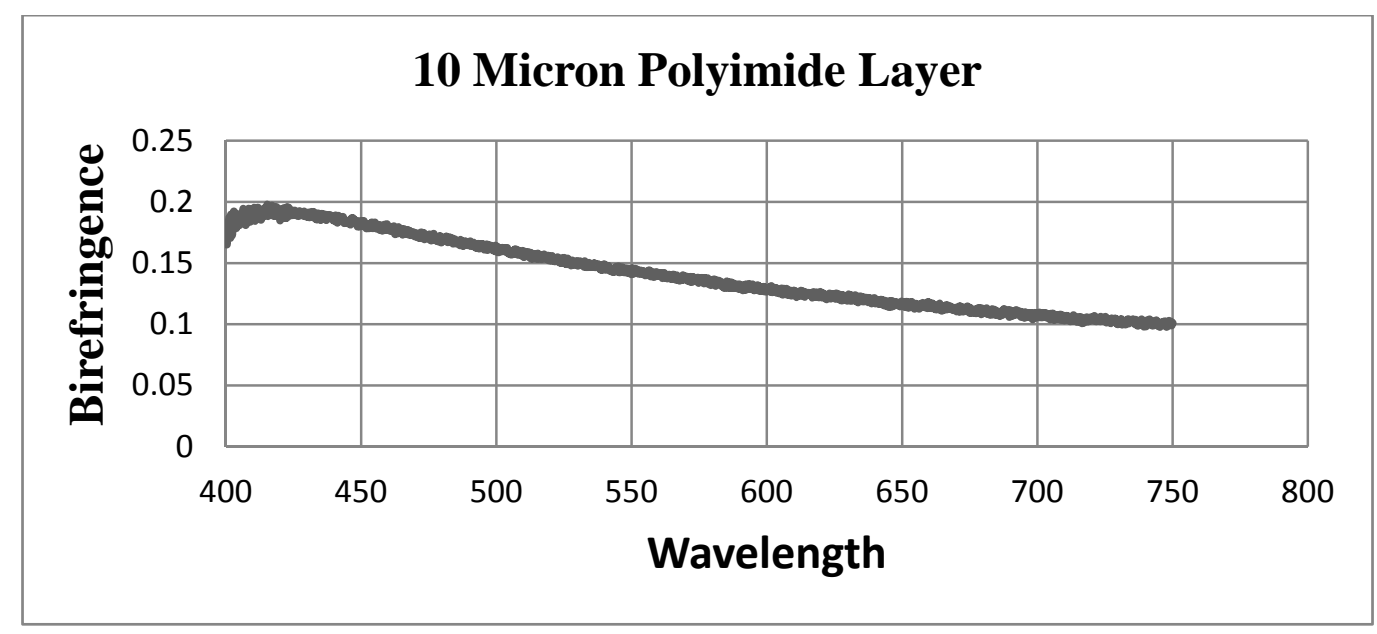

Figure 12. Representative data for uniaxial polyimide film. transmittance between crossed polarizers versus wavelength

\subsection{SUMMARY}

Adaptive optics is traditionally used to compensate for atmospheric turbulence in complex imaging systems. The inherent batch processing associated with the fabrication of new emerging phase and polarization spatial light modulators enables these technologies to be used in other applications. NASA Langley is pursuing star sensors, tunable filters, small satellite, large space telescopes for heliophysics and exoplanet coronagraph missions. This paper presented the design , fabrication and performance evaluation of phase and polarization compensators. The $1024 \times 768$ phase modulator was able to achieve the diffraction limit. The Polarization compensation offered over a 10 fold improvement to the NASA baseline $8 \mathrm{~m}$ coronagraph telescope. In addition to significantly enhancing the imagery for a wide variety of remote sensing systems the technology offers advantages in speed, size, cost, weight and radiation tolerance.

\section{REFERENCES}

[1] Clark N. "Design and Performance Evaluation of Sensors and Actuators for Advanced Optical Systems (Keynote)", SPIE Nanosensors, Biosensors, and Info-Tech Sensors and Systems 2011, Vol 7980, April 2011

[2] S.T Wu, Nematic Liquid Crystals. Spatial Light Modulator Technology- Materials, Devices and Applications, ed U. Efron, Vol 1. Marcel Dekker, Inc., New York, 1995. 
[3] Pancharatnam Proc Indian Acad. Sci A 44, 247 (1956).

[4] Yeh and M Paukshto, "Molecular crystalline thin film E-polarizer," Mol. Materials, Vol. 14, pp 1-19 (2001).

[5] Robert K Tyson "Principles of Adaptive Optics", Academic Press; 2 edition (October 13, 1997).

[6] Berraman D. "Optics in Stratified and Anisotropic Media: 4x4 Matrix Formulation, J Opt Soc. Am 502, pp502-510 April (1972).

[7] Breckinridge, James and Oppenheimer, Ben "Polarization Effects In Reflecting Coronagraphs for White-Light Applications in Astronomy", The Astrophysical Journal, 600:1091-1098, January 2004

[8] Richard G. Lyon, Mark Clampin, Peter Petrone, Patrick Thompson, Timothy Madison, Robert Woodruff, Charley Noecker, Steve Kendrick Technology Advancement of the Visible Nulling Coronagraph, International Conference in the Spirit of Lyot, Paris France, October 2010

[9] Ford, V. et al., TPF-C Flight Baseline Interim Report, http://planetquest.jpl.nasa.gov/documents/TPFC- $\quad$ FB1_Report.pdf

[10] Belikov, R. et al., Demonstration of High Contrast in 10\% Broadband Light with the Shaped Pupil Coronagraph, Proc. SPIE Vol 6693-36 (2007).

[11] Kasdin, N.J., R. J. Vanderbei, D. N. Spergel, and M. G. Littman, "Extrasolar planet finding via optimal apodized-pupil and shaped-pupil coronagraphs," The Astrophysical Journal 582, pp. 1147-1161, January (2003).

[12] Guyon, O., et al., "Exoplanet Imaging with a Phase-induced Amplitude Apodization Coronagraph. I. Principle", ApJ, 622, 744758 (2005).

[13] Breckinridge, J. B. and B. R. Oppenheimer "Polarization effects in reflecting coronagraphs for white-light applications in astronomy", Astrophys. J. 600: 1091-1098, (2004).

[14] Clark, N and Breckinridge $\mathrm{j}$ "Polarization compensation ofFresnel aberrations in telescopes", SPIE UV/Optical/IR Space Telescopes and Instruments: Innovative Technologies and Concepts, Vol 8145 Sept 2011.

[15] Brosseua, C "Fundamentals of Polarized Light: A Statistical Optics Approach", John Wiley \& Sons, 1998.

[16] Goldstein, D., "Polarized Light. Third Edition”, CRC Press 2010.

[17] Hodkinson,I and Wu, Q “ Birefringent Thin Films and Polarizing Elements” World Scientific Press, 1998.

[18] D. W. Berreman: "Optics in smoothly varying planar structures: Application to liquid-crystal twist cells", J. Opt. Soc. Am., 63, 1374-1380 (1973)

[19] Eidner, K, Mayer G., Schmidt M., and Schmiedel H., "Light propagation in stratified anisotropic media: orthoganlity and symmetry properties of the 4x4 matrix formalisms" Mol Cryst. Liq Cryst. 172, 191 (1989).

[20] Eidner K. "Light propagation in stratified anisotropic media: orthoganlity and symmetry properties of the $4 \times 4$ matrix formalisms",J. Opt. soc. Am. A 616571989

[21] Oldano C. "Electromagnetic wave propagation in Anisotropic stratified media" Phys. Rev A 40 6014-6020 (1989).

[22] Eidner, K e.a.: "Optics in stratified media - the use of optical eigenmodes of uniaxial crystals in the $4 \times 4-$ matrix formalism”, Mol. Cryst. Liq. Cryst., 172, 191-200 (1989)

[23] Hasegawa M and Taira Y 1995 Proc. 21st JLCC p 344.

[24]H. Y. Joo, H. J. Kim, S. J. Kim, and S. Y. Kim, Thin Solid Films 368, 672000.

[25] Chigrinov V, Kozenkov V, and Kwik H, "Photoalignment of Liquid Crystaline Materials", Wiley 2008. 